\title{
A Flexible Cooking Zone Composed of Partially Overlapped Inductors
}

\author{
Javier Serrano, Student Member, Jesús Acero, Member, Ignacio Lope, Member, Claudio Carretero, Senior \\ Member, and José Miguel Burdío, Senior Member
}

\begin{abstract}
Domestic induction cookers are evolving from fixed cooking areas to flexible surfaces, in such a way that the pot can be placed at any position. This implies the use of a larger number of reduced-sized inductors which present a lower efficiency. As a solution to increase the efficiency while maintaining the flexibility, we propose the use of partially overlapped inductors of a larger size. This concept is currently in use in wireless power transfer systems, where the transmitter arrangement consists of several overlapped coils. The aim of this work is to evaluate this concept applied to domestic induction heating appliances, with special emphasis in analyzing the effects of introducing the multi-coil system with dissipative media. Moreover, the losses in the winding will be studied in detail. The system will be prototyped and tested delivering up to $3.7 \mathrm{~kW}$.
\end{abstract}

Index Terms-Domestic induction heating, electromagnetic analysis, multi-coil system, winding losses.

\section{INTRODUCTION}

D OMESTIC induction heating appliances base their operation on forcing an alternating current in the range of tens of kilohertz into flowing through an inductor [1]. This generates a magnetic field of the same frequency that reaches the base of the vessel, which has to be made of an electrical conductor so that currents are induced due to Faraday's law. The induced currents produce power dissipation heating up the material. Additionally, if the material is ferromagnetic, magnetic hysteresis produce extra dissipation.

Induction heating ( $\mathrm{IH}$ ) has, in the last decades, gradually substituted other technologies as electric-resistive stoves or gas burners. The strength of induction heating technology lies on its rapidness, safety and energetic efficiency. Moreover, upper end cookers feature coil arrays, in such way, that the vessel can be placed at different positions [2]. In these cases, the most aligned inductors are activated by using a pot

Manuscript received July, 2017; revised August, 2017; accepted January 2018. This work was partly supported by the Spanish MINECO under Project TEC2016-78358-R, and Project RTC-2014-1847-6, by the Torres Quevedo Grant PTQ-14-06713, by the DGA-FSE, by the University of Zaragoza under Project JIUZ-2016-CIE-01, and by the BSH Home Appliances Group.

Javier Serrano, Jesús Acero and José Miguel Burdío are with the Department of Electronic Engineering and Communications, University of Zaragoza, Zaragoza, 50018, Spain. (e-mail: jserrano@unizar.com). Ignacio Lope is with BSH Home Appliances, Zaragoza, 50016, Spain. Claudio Carretero is with the Applied Physics, University of Zaragoza, Zaragoza, 50009, Spain.

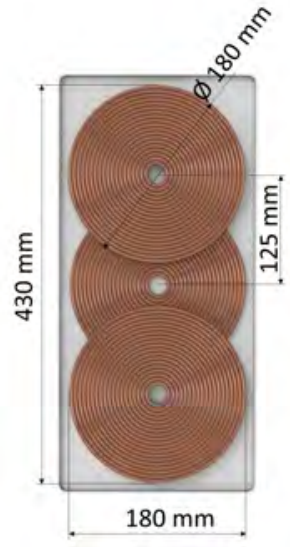

(a)

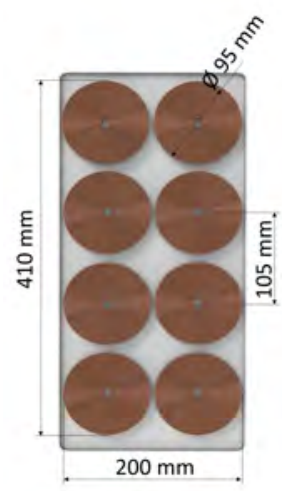

(b)
Fig. 1: Flexible cooking areas. (a) Cooking surface formed by 8 reduced-sized coils. (b) Proposed equivalent cooking surface formed by 3 large overlapped coils.

detection system. Cookers with coil arrays present flexibility as a differentiating factor while the increased number of coils makes production cost the main drawback. Additionally, to achieve flexibility, small size inductors have to be used. Efficiency was proven [3] to depend on the ratio between the coil diameter and the distance between the coil and the pot, $D_{\text {coil }} / d_{\text {pot. }}$. Therefore, the use of small inductors incurs an efficiency penalty.

In this work, we propose partially overlapped coils as a solution to increase efficiency. This allows the use of larger inductors which improve the $D_{\text {coil }} / d_{\text {pot }}$ ratio, and therefore the induction efficiency. Moreover the total number of inductors is reduced, leading to savings in the production stage while maintaining the flexibility. In this case we will focus in the modeling, design and implementation of a rectangular cooking zone of $430 \mathrm{~mm}$ x $180 \mathrm{~mm}$. Fig. 1 shows in the left side (a), one of the configurations currently commercialized. This configuration makes use of 8 adjacent coils [4]. On the right side (b), our proposal is represented. The proposed system consists of 3 partially overlapped coils which have a significantly larger diameter. Consequently, the aimed power is greater and the inductor is designed accordingly, with less turns and more strands. The main features of the presented systems can be compared in Table I. 


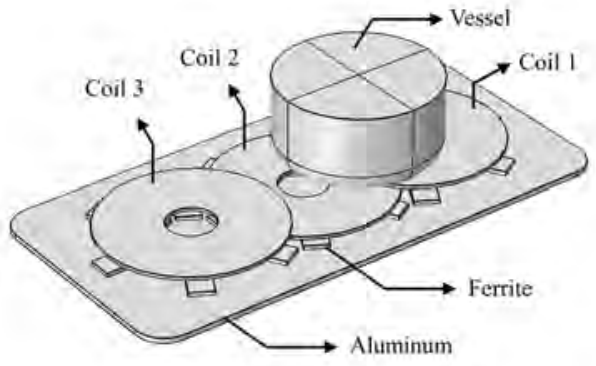

Fig. 2: Simulation model with labeled components.

\begin{tabular}{lll} 
& 8 Adjacent coils & 3 Overlapped coils \\
\hline Maximum power per coil & $500 \mathrm{~W}$ & $3700 \mathrm{~W}$ \\
Coil diameter & $95 \mathrm{~mm}$ & $180 \mathrm{~mm}$ \\
Distance between centers & $105 \mathrm{~mm}$ & $125 \mathrm{~mm}$ \\
Number of turns & 50 & 19 \\
Number of strands & 28 & 140 \\
Strand diameter & $0.2 \mathrm{~mm}$ & $0.2 \mathrm{~mm}$ \\
\hline \hline
\end{tabular}

TABLE I: Main features of reduced-sized coils and the proposed inductors.

Overlapped coils have been proven to provide good results in other applications as wireless power transfer (WPT) systems [5]-[9]. Encouraged by these results, the aim of this paper is to evaluate the performance of the solution when applied to domestic induction heating. This entails several challenges: Firstly, the overlapped coils are placed under dissipative media. What this produces is that the impedance of the inductors depends on this media, which may vary depending on the size and material of the pot, as well as frequency. Moreover, the coupling conditions of the inductors also depend on the vessel. Second, the rated power reaches $3.7 \mathrm{~kW}$, this makes necessary an intensive study of the losses in the windings to prevent overheating. Finally, the system is aimed to produce an even temperature in the base of the pot. Therefore, the power density distribution will be studied considering the coupling between coils.

The reminder of this paper is organized as follows: After this introduction, the model of coupled inductors in dissipative media will be presented. This model will be oriented to FEM simulation for design purposes and includes the extraction of the equivalent impedance of the system, the prediction of the currents through the inductors, the analysis of the power distribution in the load, the power losses in the windings and the system's global efficiency. In the next section, these aspects will be studied experimentally, verifying the model and providing useful information to evaluate the viability of the concept. Finally, conclusions will be drawn.

\section{MULTI-COIL SYSTEMS IN DISSIPATIVE MEDIA}

\section{A. Impedance matrix}

Domestic induction systems are composed of four main elements: the inductor in charge of generating the magnetic field, the ferrites which act as flux concentrator, the vessel which is the piece to heat up, and an aluminum tray, which acts as shielding. The inductor is supplied by a power converter. The impedance that the inductor presents to the converter is given by the coupling between the coil and the surrounding media.

In the case of a multi-coil arrangement, the system is commonly described in frequency domain by its impedance matrix $\mathbf{Z}(h \omega)$ [10], [11], which relates the voltage drops in the coils with the currents through them, being $\omega$ the angular frequency and $h$ the harmonic number. The impedance matrix contains the self-impedances of the inductors in the diagonal elements and coupling impedances in the non-diagonal elements. The self-impedance, $Z_{i i}(h \omega)$ of coil $i$ defines the relationship between the current and the voltage when no current is flowing through other inductors. On the other hand, the coupling impedance $Z_{i j}(h \omega)$ defines the induced voltage in coil $i$ by a current flowing through coil $j$. These relationships are represented as:

$$
\mathbf{V}(h \omega)=\mathbf{Z}(h \omega) \cdot \mathbf{I}(h \omega),
$$

where $\mathbf{V}(h \omega)$ is the column vector containing the voltage drop in each coil $\left\{V_{1}(h \omega), V_{2}(h \omega) \ldots V_{n}(h \omega)\right\}$ and $\mathbf{I}(h \omega)$ is the column vector containing the current through each of the coils $\left\{I_{1}(h \omega), I_{2}(h \omega) \ldots I_{n}(h \omega)\right\}$.

Fig. 2 shows the developed FEM model with labeled components and numbered coils. The simulation model consists of the following elements: three coils, one aluminum tray, ferrites and the vessel. The inductors are modeled by ring-shaped current densities flowing through a non-dissipative media [12]. As the coils are modeled by an ideal material with no losses, their actual losses are calculated afterwards by means of a specific loss model (Section D). The dissipative elements in the model are the vessel, which is made of ferromagnetic steel, and the shielding tray which is usually made of aluminum. Both are characterized in the simulation model by their electrical conductivity and their relative magnetic permeability. The ferrite (3C90) is characterized by its permeability and a null electrical conductivity. At the frequency and magnetic flux operation range, the losses in the ferrite are very low and can be neglected [13].

A three-coil system as the one proposed in this work, can be described by a $3 \times 3$ impedance matrix (omitting the frequency dependence in the notation for the sake of clarity) as:

$$
\left[\begin{array}{l}
V_{1} \\
V_{2} \\
V_{3}
\end{array}\right]=\left[\begin{array}{lll}
Z_{11} & Z_{12} & Z_{13} \\
Z_{21} & Z_{22} & Z_{23} \\
Z_{31} & Z_{32} & Z_{33}
\end{array}\right]\left[\begin{array}{l}
I_{1} \\
I_{2} \\
I_{3}
\end{array}\right]
$$

However, the problem is fully symmetric with respect to the central coil. This fact leads to some simplifications: Firstly, the coils located at both ends are identical $Z_{11}=Z_{33}$, and the coupling between them is negligible $Z_{13}=Z_{31}=0$. In the second place, the coupling between coils $1-2$ and coils $2-3$ 


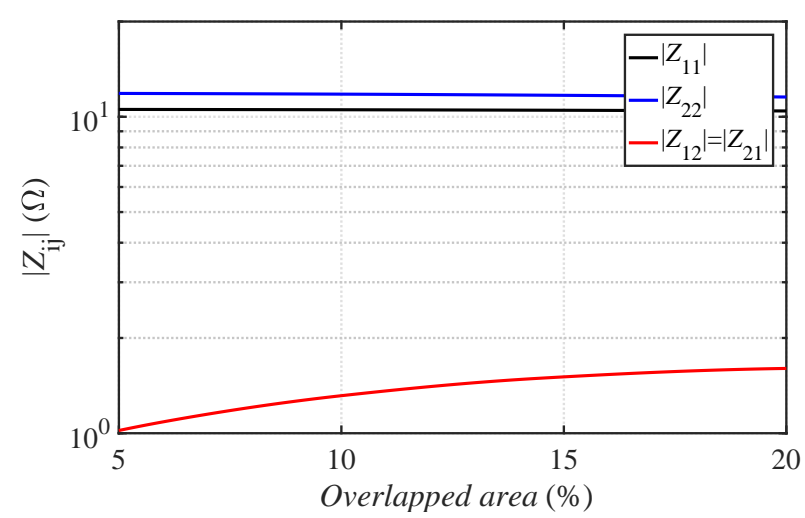

Fig. 3: Norm of the terms of the impedance matrix as a function of the overlapping area between two inductors (40 $\mathrm{kHz})$.

are equal, leading to $Z_{12}=Z_{23}$ and $Z_{21}=Z_{32}$. Therefore, the matrix can be simplified to:

$$
\left[\begin{array}{l}
V_{1} \\
V_{2} \\
V_{3}
\end{array}\right]=\left[\begin{array}{ccc}
Z_{11} & Z_{12} & 0 \\
Z_{21} & Z_{22} & Z_{21} \\
0 & Z_{12} & Z_{11}
\end{array}\right]\left[\begin{array}{c}
I_{1} \\
I_{2} \\
I_{3}
\end{array}\right]
$$

Since obtaining $Z_{11}, Z_{22}, Z_{12}$ and $Z_{21}$ is sufficient to describe the whole system, we will focus the analysis on two coils. The matrix equation for coils 1 and 2 is given by:

$$
\left[\begin{array}{l}
V_{1} \\
V_{2}
\end{array}\right]=\left[\begin{array}{ll}
Z_{11} & Z_{12} \\
Z_{21} & Z_{22}
\end{array}\right]\left[\begin{array}{c}
I_{1} \\
I_{2}
\end{array}\right]
$$

According to the matrix system eq. (4), two simulations need to be carried out. In the first iteration, coil 1 is excited with a current $I_{1}$ of $1 \mathrm{~A}$. Then, the voltage drop of each coil $\left\{V_{1}, V_{2}\right\}$ is calculated as the line integral of the electric field along the cable's trajectory [12]. Once the voltages are known, the first column of the impedance matrix, $\left\{Z_{11}, Z_{21}\right\}$, is obtained by dividing the voltages by the causing current. The second column of the impedance matrix is obtained analogously by exciting coil 2 .

The term $Z_{11}$ relates the current through coil 1 to the voltage drop across it when coil 2 is turned off. Analogously, $Z_{22}$ relates the current through coil 2 to its voltage drop. These are independent of the relative position of the coils. On the other hand, the coupling impedances $Z_{12}$ and $Z_{21}$ relate the current flowing through one coil to the voltage induced in the other. These are largely dependent on the relative positions between the coils, as can be seen in Fig. 3, which shows the terms of the impedance matrix with respect to the overlapping area.

The distance between coil centers has been set to $125 \mathrm{~mm}$ so that 16 identical ferrite bars can be used, producing a symmetrical ferrite layout. This fixes the overlapping area to a $19.3 \%$ of the inductor area. The coils in the sides have a distance of $4 \mathrm{~mm}$ to the vessel while the coil in the middle is $6 \mathrm{~mm}$ away from the pot.

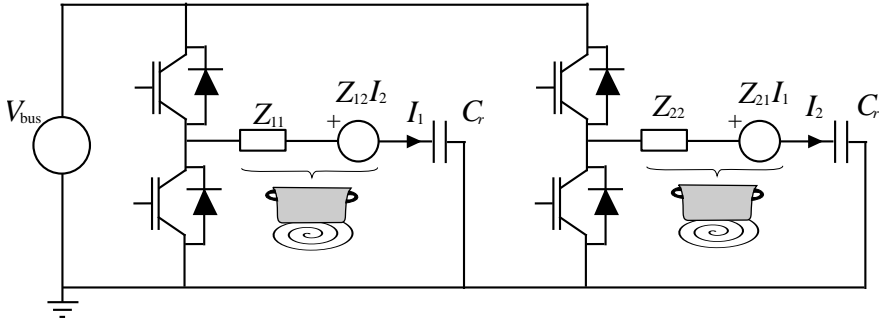

Fig. 4: Schematic of two half-bridge inverters powered by a common de bus with two coupled induction loads.

\section{B. Current through the inductors}

Once the impedance matrix describing the system has been obtained, the currents through each coil can be predicted. The current through the coils is given by the matrix impedance and the converter topology. In this case, we will make use of two half-bridge inverters with series resonant load as represented in Fig. 4, where $V_{\text {bus }}$ is a dc voltage, $V_{o, 1}$ and $V_{o, 2}$ are the output voltages of the half-bridges, and $C_{r}$ is the resonant capacitor, which is assumed identical in both cases.

With this topology, eq. (1) can be rewritten as:

$$
\mathbf{V}_{\mathbf{o}}=\left(\mathbf{Z}+\mathbf{Z}_{C r}\right) \cdot \mathbf{I},
$$

being $\mathbf{V}_{\mathbf{o}}$ the column vector containing the output voltages of each inverter $\left\{V_{o, 1}, V_{o, 2}\right\}$ and $\mathbf{Z}_{C r}$ the matrix containing the impedance of the resonant capacitors: $Z_{C r}=-1 /\left(h \omega C_{r}\right)$ in the diagonal terms and zeros in the crossed terms.

Solving eq. (5) the solutions for the currents are obtained:

$I_{1}(t)=\sum_{h=1}^{n} \frac{V_{o, 1}\left(Z_{22}+Z_{C r}\right)-V_{o, 2} Z_{12}}{Z_{11} Z_{22}-Z_{12} Z_{21}+\left(Z_{11}+Z_{22}\right) Z_{C r}+Z_{C r}{ }^{2}}$,

$I_{2}(t)=\sum_{h=1}^{n} \frac{V_{o, 2}\left(Z_{11}+Z_{C r}\right)-V_{o, 1} Z_{21}}{Z_{22} Z_{11}-Z_{21} Z_{12}+\left(Z_{22}+Z_{11}\right) Z_{C r}+Z_{C r}{ }^{2}}$,

where the impedance terms $Z_{i j}=Z_{i j}(h \omega)$ are the impedance of the $h^{t h}$ harmonic, and $V_{o, i}$ is a rectangular waveform whose harmonic decomposition is given by

$$
V_{o, i}=\frac{2 V_{\text {bus }}}{h \pi} \sin \left(h \pi D_{i}\right) e^{j\left(\tan ^{-1}\left(\frac{1}{\tan \left(h \pi D_{i}\right)}\right)+h \phi_{v, i}\right)}
$$

as a function of the voltages phase-shift $\phi_{v, i}$ and the duty cycle of each inverter $D_{i}$.

The current through the inductors is of great importance, not only for affecting the converter design, but also for determining the power dissipation in each element (vessel, aluminum and winding) as will be seen in the following sections. 


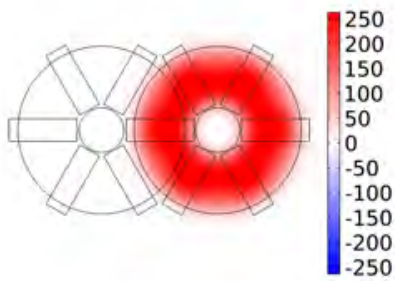

(a)

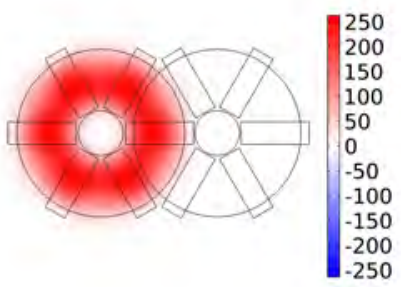

(b)
Fig. 5: Diagonal elements of $\overline{\mathbf{S}}\left(\Omega / \mathrm{m}^{2}\right)$ representing the power density caused by each coil independently. $\bar{E}_{x, 1} \bar{H}_{y, 1}^{*}-\bar{E}_{y, 1} \bar{H}_{x, 1}^{*}$. (b) $\hat{E}_{x, 2} \bar{H}_{y, 2}^{*}-\bar{E}_{y, 2} \hat{H}_{x, 2}^{*}$. (a)

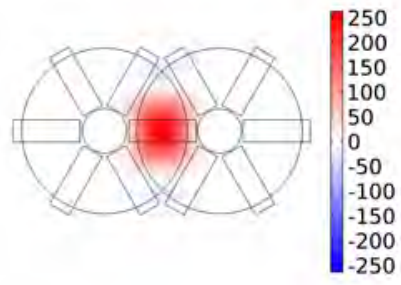

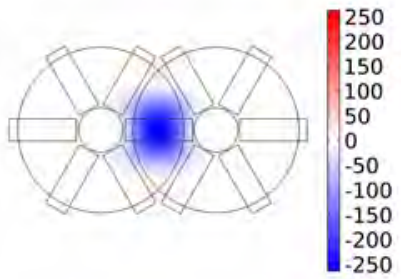

(b)
Fig. 6: Non-diagonal elements of $\overline{\mathbf{S}}\left(\Omega / \mathrm{m}^{2}\right)$ $\bar{E}_{x, 1} \bar{H}_{y, 2}^{*}-\bar{E}_{y, 1} \bar{H}_{x, 2}^{*}=\bar{E}_{x, 2} \bar{H}_{y, 1}^{*}-E_{y, 2} \bar{H}_{x, 1}^{*}$ representing the power density caused by the coupled fields. (a) Inductors in phase. (b) Inductors in opposing-phase.

\section{Surface power density}

The power density in the surface of a dissipative medium, $\rho_{\text {surf }}(\omega h)$, is given by the Poynting vector's flux through the surface as [14]:

$$
\begin{aligned}
p_{\text {surf }}(\omega h)=\operatorname{Re}( & \mathbf{S}(\omega h) \cdot \widehat{\mathbf{n}}) \\
= & \operatorname{Re}\left(\frac{1}{2}\left(\mathbf{E}(\omega h) \times \mathbf{H}^{*}(\omega h)\right) \cdot \widehat{\mathbf{n}}\right)
\end{aligned}
$$

where $\hat{\mathbf{n}}$ is the normal unitary vector of the surface, and $\mathbf{E}$ and $\mathbf{H}$ are the total electric and magnetic fields respectively, which in the case of arrangements with $n_{c}$ coils and assuming linear media, can be calculated applying superposition [15]:

$$
\begin{aligned}
\mathbf{E}(\omega h) & =\sum_{i=1}^{n_{c}} I_{i} \overline{\mathbf{E}}_{i}(\omega h), \\
\mathbf{H}(\omega h) & =\sum_{i=1}^{n_{c}} I_{i} \overline{\mathbf{H}}_{i}(\omega h) .
\end{aligned}
$$

being $\overline{\mathbf{E}}$ and $\overline{\mathbf{H}}$ the fields per unit of current and $I_{i}$ the solutions of eq. (5) for the $i^{t h}$ coil.

In the model, there are two main elements with the sufficient electrical conductivity to produce a significant amount of losses. The pot is meant to dissipate most of the power, while shielding elements as aluminum produce

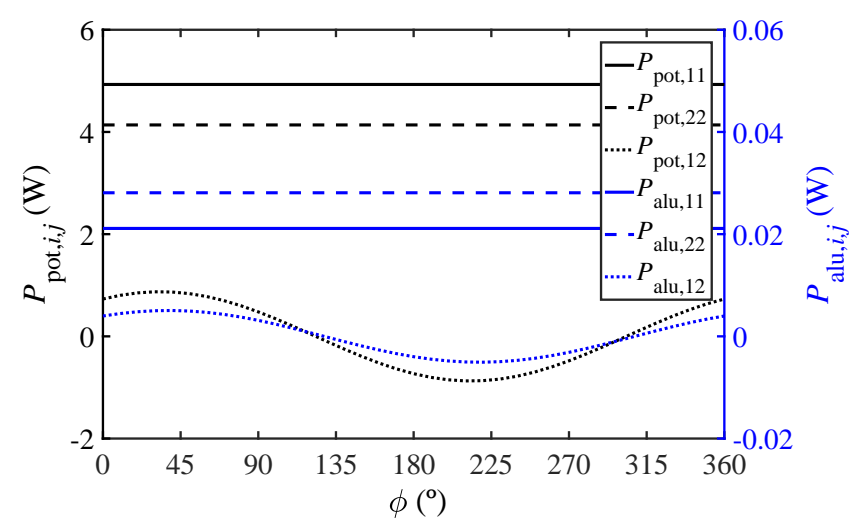

Fig. 7: Decomposition of the dissipated power when each inductor drives a current of $1 \mathrm{~A}$ and their phase-shift is $\phi$. Black color for the components of $P_{\text {pot }}$ and blue for the components of $P_{\mathrm{alu}}$.

undesired losses. Both of them are placed horizontally, and therefore, their normal vector $\hat{\mathbf{n}}_{\text {surf }}$ is coincident with the $\hat{\mathbf{z}}$ axis. As a consequence, only the $\hat{\mathbf{z}}$ component of the Poynting vector produces power.

Combining (9), (10) and (11) the power density in the surface of a dissipative medium can be expressed as:

$$
p_{\text {surf }}=\operatorname{Re}\left(\mathbf{I}^{*} \cdot \overline{\mathbf{S}} \cdot \mathbf{I}\right)
$$

which can be particularized for two inductors causing dissipation on a vessel with a horizontal base as:

$$
\begin{aligned}
& \mathbf{I}=\left[\begin{array}{l}
I_{1} \\
I_{2}
\end{array}\right] \\
& \overline{\mathbf{S}}=\left[\begin{array}{cc}
\bar{E}_{x, 1} \bar{H}_{y, 1}^{*}-\bar{E}_{y, 1} \bar{H}_{x, 1}^{*} & \bar{E}_{x, 1} \bar{H}_{y, 2}^{*}-\bar{E}_{y, 1} \bar{H}_{x, 2}^{*} \\
\bar{E}_{x, 2} \bar{H}_{y, 1}^{*}-E_{y, 2} \bar{H}_{x, 1}^{*} & \hat{E}_{x, 2} \bar{H}_{y, 2}^{*}-\bar{E}_{y, 2} \hat{H}_{x, 2}^{*}
\end{array}\right] .
\end{aligned}
$$

Fig. 5 shows the results for the diagonal elements of the matrix $\overline{\mathbf{S}}$ when the pot fully covers the inductors, while Fig. 6 depicts the non-diagonal elements when currents are in phase and opposing-phase.

As can be derived from eq. (14), diagonal elements of $\overline{\mathbf{S}}$ represent the power dissipation in the surface generated by $1 \mathrm{~A}$ circulating through coils 1 and 2 respectively. The result only depends on the electric and magnetic field generated by each of the coils, and therefore, they are independent of the operation conditions of the other inductor. On the other hand, nondiagonal elements represent the power dissipation produced by the coupled fields. In this case, power density depends on the phase-shift between the currents. By integrating the surface power density in the surface of the pot $S_{p o t}$ the power, $P_{p o t}$ is obtained.

$$
P_{\mathrm{pot}}=\iint_{S_{\mathrm{pot}}} \operatorname{Re}\left(\mathbf{I}^{*} \cdot \overline{\mathbf{S}} \cdot \mathbf{I}\right) d S_{\mathrm{pot}}
$$

which for the case of two coils can be expressed as in eq. (16). 


$$
P_{\mathrm{pot}}=\underbrace{\operatorname{Re}\left(I_{1} I_{1}^{*} \iint_{S_{\mathrm{pot}}} S_{11} d S_{\mathrm{pot}}\right)}_{P_{\mathrm{pot}, 11}}+\underbrace{\operatorname{Re}\left(I_{2} I_{2}^{*} \iint_{S_{\mathrm{pot}}} S_{22} d S_{\mathrm{pot}}\right)}_{P_{\mathrm{pot}, 22}}+\underbrace{\operatorname{Re}\left(I_{1} I_{2}^{*} \iint_{S_{\mathrm{pot}}} S_{12} d S_{\mathrm{pot}}\right)}_{P_{\mathrm{pot}, 12}}+\underbrace{\operatorname{Re}\left(I_{2} I_{1}^{*} \iint_{S_{\mathrm{pot}}} S_{21} d S_{\mathrm{pot}}\right)}_{P_{\mathrm{pot}, 21}}
$$

Naturally, the same reasoning applies also to the aluminum, which has a surface $S_{\text {alu }}$ and dissipates a power $P_{\text {alu }}$. These terms are represented in Fig. 7 when both $I_{1}$ and $I_{2}$ have an amplitude of $1 \mathrm{~A}$ and a phase-shift $\phi$.

\section{Winding losses}

Inductors for domestic IH are usually wound of litz wire, a multi-stranded cable with a twisting pattern which, ideally, make all strands equivalent among them favoring the equal current sharing. The losses can be divided into two groups depending on their origin: conduction losses and proximity losses [16]-[19]. Conduction losses are those produced when a current caused by an external voltage source flows through a material with a certain electrical conductivity. This includes $\mathrm{dc}$ losses and skin effect. On the other hand, proximity losses are generated by induced currents caused by the alternating magnetic field generated by itself or by neighboring coils.

Losses in one single winding are commonly represented as a resistance $R_{w}$ which includes both conduction resistance and $R_{\text {cond }}$ proximity resistance $R_{\text {prox }}$. In accordance, power losses in the winding are given by;

$$
P_{w}=R_{w} I^{2}=\left(R_{\text {cond }}+R_{\text {prox }}\right) I^{2}
$$

Expressions for these resistances are found in literature [20] as a function of the parameters of the inductor and the cabling structure: strand radius $r_{s}$, electrical conductivity of the cable $\sigma_{w}$, number of turns $n_{t}$ and number of strands $n_{s}$. Additionally, $l_{a v}$ is the average length of the turns and can be calculated from the external and internal radii as $\pi\left(r_{\mathrm{ext}}+r_{\mathrm{int}}\right)$.

$$
R_{\text {cond }}=\frac{n_{t} l_{a v}}{\sigma_{w} n_{s} \pi r_{s}^{2}} \Phi_{\text {cond }}\left(\frac{r_{s}}{\delta}\right)^{\frac{r_{s}}{\delta}} \approx<1 \frac{n_{t} l_{a v}}{\sigma_{w} n_{s} \pi r_{s}^{2}}
$$

In the expression above, $\Phi_{\text {cond }}\left(\frac{r_{s}}{\delta}\right)$ is a function which introduces the effect of frequency through the skin depth $\delta$. However, the coils are designed so that the strand radius is smaller than the skin depth. Under such circumstance, commonly referred as low frequency regime, $\Phi_{\text {cond }}\left(\frac{r_{s}}{\delta}\right)$ takes a unitary value, and $R_{\text {cond }}$ is reduced to the dc resistance.

On the other hand, the proximity resistance is given by [20]:

$$
\begin{aligned}
R_{\text {prox }}=n_{t} n_{s} \frac{4 \pi}{\sigma_{w}} \Phi_{\text {prox }}\left(\frac{r_{s}}{\delta}\right)\left\langle\overline{\mathbf{H}}_{t} \cdot \overline{\mathbf{H}}_{t}^{*}\right\rangle_{V_{\text {coil }}} \ldots \\
\stackrel{r_{s}}{\frac{r_{s}}{\delta}} \stackrel{<1}{\approx} n_{t} n_{s} \frac{4 \pi}{\sigma_{w}}\left(\frac{r_{s}}{\delta}\right)^{4}\left\langle\overline{\mathbf{H}}_{t} \cdot \overline{\mathbf{H}}_{t}^{*}\right\rangle_{V_{\text {coil }}}
\end{aligned}
$$

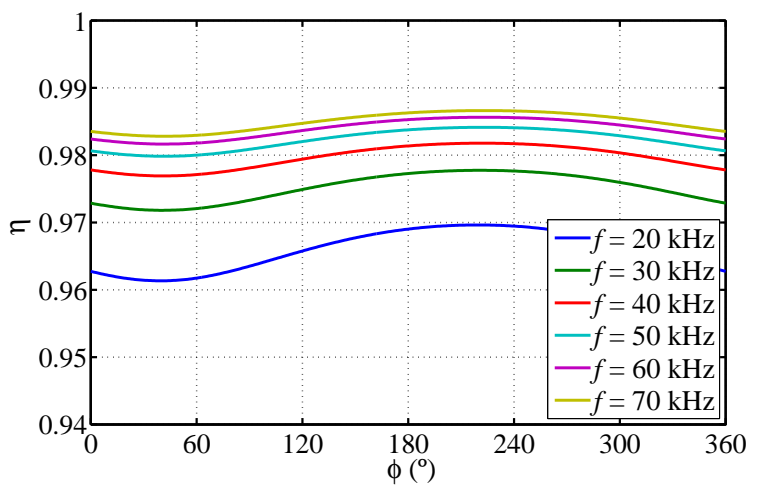

Fig. 8: First harmonic efficiency of two fully covered inductors activated simultaneously as a function of phase-shift and operating frequency.

where $\Phi_{\text {prox }}\left(\frac{r_{s}}{\delta}\right)$ introduces the frequency dependence and can be approximated in low frequency regime by $\left(\frac{r_{s}}{\delta}\right)^{4}$. Additionally, $\overline{\mathbf{H}}_{t}$ is the magnetic field per unit of current which is transversal to the conductors, and the \langle\rangle$_{V_{\text {coil }}}$ operator represents the average in the volume of the coil $V_{\text {coil }}$. Note that, in the case of single-inductor arrangements, the coil presents cylindrical symmetry and therefore the magnetic field is always transversal to the conductors.

When two coils are driving current the conduction losses in these inductors are given by

$$
P_{\text {cond }}=\left[\begin{array}{cc}
I_{1}^{*} & I_{2}^{*}
\end{array}\right]\left[\begin{array}{cc}
\frac{n_{t} l_{a v}}{\sigma_{w} n_{s} \pi r_{s}^{2}} & 0 \\
0 & \frac{n_{t} l_{a v}}{\sigma_{w} n_{s} \pi r_{s}^{2}}
\end{array}\right]\left[\begin{array}{c}
I_{1} \\
I_{2}
\end{array}\right],
$$

or equivalently,

$$
P_{\text {cond }}=\frac{n_{t} l_{a v}}{\sigma_{w} n_{s} \pi r_{s}^{2}}\left(I_{1}^{2}+I_{2}^{2}\right)
$$

With respect to proximity losses [21], unlike in single-coils systems, there is no cylindrical symmetry, and therefore, conductors are subject to both transversal fields and longitudinal fields. The losses caused by transversal fields on the three coils when two coils are activated are given by:

$$
\begin{aligned}
& P_{\text {prox }, t}=n_{t} n_{s} \frac{4 \pi}{\sigma_{w}}\left(\frac{r_{s}}{\delta}\right)^{4}\left[\begin{array}{ll}
I_{1}^{*} & I_{2}^{*}
\end{array}\right] \ldots \\
& \cdot \sum_{k=1}^{3}\left[\begin{array}{ll}
\left\langle\overline{\mathbf{H}}_{t, 1} \cdot \overline{\mathbf{H}}_{t, 1}^{*}\right\rangle_{V_{\text {coil, } \mathrm{k}}} & \left\langle\overline{\mathbf{H}}_{t, 1} \cdot \overline{\mathbf{H}}_{t, 2}^{*}\right\rangle_{V_{\text {coil, }, \mathrm{k}}} \\
\left\langle\overline{\mathbf{H}}_{t, 2} \cdot \overline{\mathbf{H}}_{t, 1}^{*}\right\rangle_{V_{\text {coil, }, \mathrm{k}}} & \left\langle\overline{\mathbf{H}}_{t, 2} \cdot \overline{\mathbf{H}}_{t, 2}^{*}\right\rangle_{V_{\text {coil }, \mathrm{k}}}
\end{array}\right]\left[\begin{array}{c}
I_{1} \\
I_{2}
\end{array}\right] .
\end{aligned}
$$

Losses caused by fields which are longitudinal to the conductors, were proven to produce half of the losses than 
the same field striking transversally [22], and therefore can be calculated as:

$$
\begin{aligned}
& P_{\text {prox }, l}=n_{t} n_{s} \frac{2 \pi}{\sigma_{w}}\left(\frac{r_{s}}{\delta}\right)^{4}\left[\begin{array}{cc}
I_{1}^{*} & I_{2}^{*}
\end{array}\right] \ldots \\
& \cdot \sum_{k=1}^{3}\left[\begin{array}{ll}
\left\langle\overline{\mathbf{H}}_{l, 1} \cdot \overline{\mathbf{H}}_{l, 1}^{*}\right\rangle_{V_{\text {coil, }, \mathrm{k}}} & \left\langle\overline{\mathbf{H}}_{l, 1} \cdot \overline{\mathbf{H}}_{l, 2}^{*}\right\rangle_{V_{\text {coil, } \mathrm{k}}} \\
\left\langle\overline{\mathbf{H}}_{l, 2} \cdot \overline{\mathbf{H}}_{l, 1}^{*}\right\rangle_{V_{\text {coil, } \mathrm{k}}} & \left\langle\overline{\mathbf{H}}_{l, 2} \cdot \overline{\mathbf{H}}_{l, 2}^{*}\right\rangle_{V_{\text {coil, } \mathrm{k}}}
\end{array}\right]\left[\begin{array}{c}
I_{1} \\
I_{2}
\end{array}\right],
\end{aligned}
$$

\section{E. Efficiency}

The energetic efficiency of the system can be obtained from the previous calculations. This key performance parameter can be defined, as usual, as the ratio between useful output power and input power, which applied to domestic IH appliances, translates into power dissipated in the vessel divided by total power (including losses in windings and aluminum). As the intervening terms depend on both frequency and phase-shift, the efficiency also depends on these variables.

$$
\eta=\frac{P_{\mathrm{pot}}}{P_{\mathrm{pot}}+P_{\mathrm{alu}}+P_{\mathrm{cond}}+P_{\mathrm{prox}, t}+P_{\mathrm{prox}, l}}
$$

The terms of eq. (24) are given by eq. (16) applied to the pot and the aluminum, and eq. (21), (22), (23) for the losses in the windings.

Fig. 8 shows the calculated efficiency when two fully covered inductors are activated simultaneously carrying the same current. The obtained efficiencies range from 97.2 $\%$ to $98.6 \%$ in the operating frequencies $(30 \mathrm{kHz}$ to 70 $\mathrm{kHz}$ ) which is a significant improvement with respect to the efficiency presented by smaller inductors currently used in flexible induction surfaces, which is roughly $96 \%$ [4] when fully covered. This implies that losses are reduced approximately by half.

Although the efficiency of fully covered inductors is commonly used as figure of merit, free induction surfaces have the particularity of providing the user the chance to place the pot in any position. As a consequence, under real working conditions, the coils might be partially uncovered. Therefore, it is of interest to evaluate the efficiency of the systems when using a circular vessel of a representative size $(20 \mathrm{~cm})$ placed at different positions. This is represented in Fig. 9. The results correspond to a frequency of $40 \mathrm{kHz}$ and when two coils are simultaneously activated, the phase-shift is set to $120^{\circ}$ as that results in a good heat distribution. This results show how the maximum efficiency (dashed line) is achieved with only one coil, however, to obtain a good heat distribution, two coils need to be activated when the vessel is placed between them. This reduces the efficiency at some positions due to the use of two partially uncovered coils. This efficiency corresponds to the optimal performance in global terms and is represented in circles. The average efficiency, considering that two coils have to be activated at the same time to achieve the optimal performance, is $95.99 \%$. This implies an improvement with

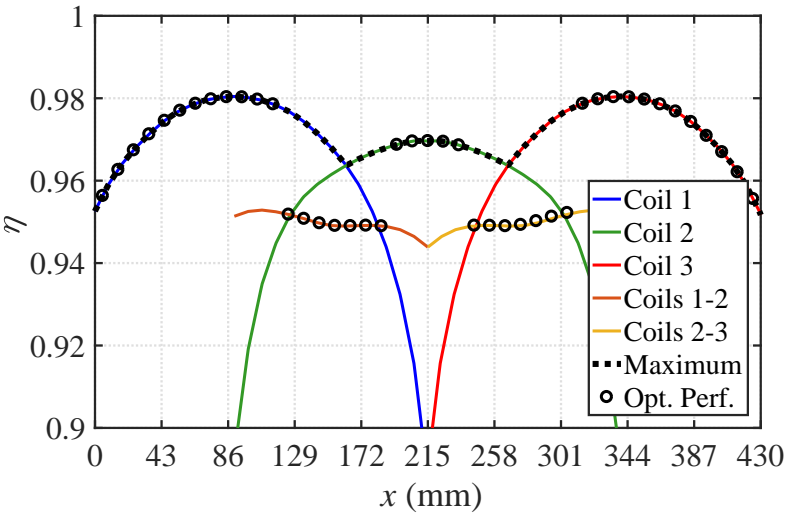

(a)

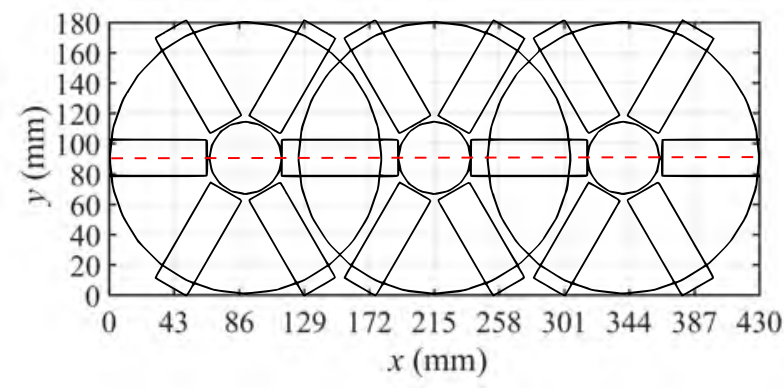

(b)

Fig. 9: First harmonic efficiency when a $20 \mathrm{~cm}$ pot is placed at different positions along the central axis. The $x$ axis represents where the center of the vessel is placed. (a) Simulated efficiency at $40 \mathrm{kHz}$. (b) Top view of the system showing in red the simulated positions.

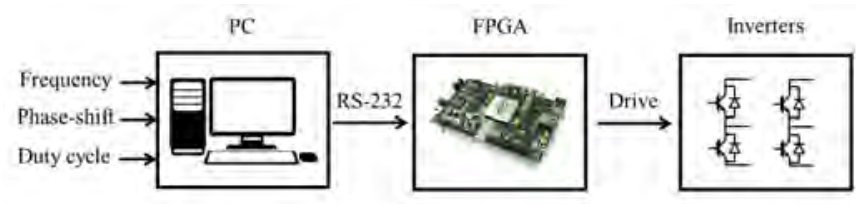

Fig. 10: Open loop modulation scheme.

respect to the small adjacent coils, which have an efficiency of $96 \%$ when completely covered and a significantly lower efficiency when partially uncovered.

\section{EXPERIMENTAL RESULTS}

The experimental setup built for the evaluation of the concept consists on two synchronized half-bridge inverters. Each of them is based on the inverter module presented in [23]. The modulation is done in open loop by an FPGA which receives the instructions from a PC interface. This is represented in Fig. 10. The main voltage and current waveforms were acquired by means of a Tektronix DPO 7354 Oscilloscope with Pearson Model-110 current monitors and Tektronix P5205 differential probes. Fig. 11 shows the complete setup and the built prototype is shown in better detail in Fig. 12. 


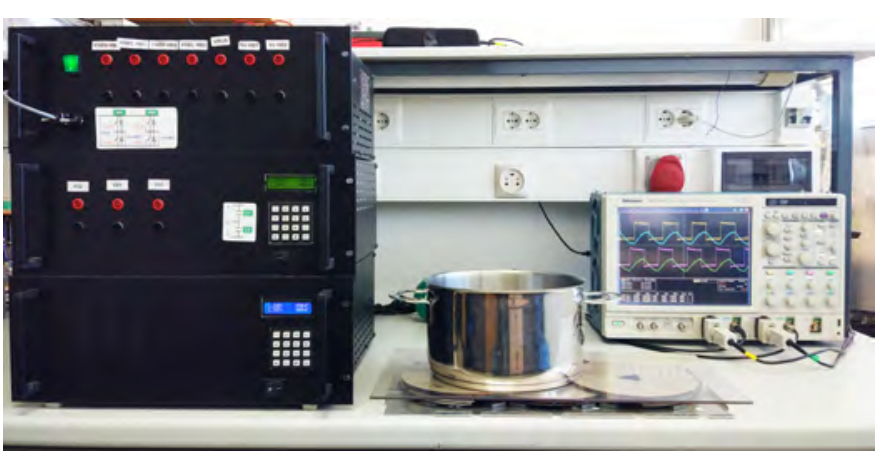

Fig. 11: Experimental setup.

In order to test the simultaneous activation of two inductors, a vessel made of a ferromagnetic steel was placed in the geometric center of the two coils under study. As a consequence, both inductors were partly covered by the pot. This scenario is shown in Fig. 12(b) and will be the case study in the following experiments.

\section{A. Impedance matrix}

Firstly, the calculated impedance matrix will be verified. As seen before, the impedance $Z_{i j}$ defines the relationship between the current $I_{j}$ and the induced voltage in coil $i$. Due to the resistive-inductive character of the elements in the impedance matrix, they are commonly expressed as the series connection of a resistance and an inductance is such a way that

$$
Z_{i j}=R_{i j}+j h \omega L_{i j}
$$

Fig. 13 shows the simulated and measured first harmonic impedances ( $h=1$ ) defining the system. The measured impedances have been obtained as the ratio between the first harmonic component of the voltage in coil $i$ and the first harmonic component of the current in coil $j$ when $j$ is the only active coil and the rest of the coils are in open circuit. The tests were ran at real working conditions (up to $3.7 \mathrm{~kW}$ ) throwing consistent results.

Higher harmonics are of lower interest, as typical $\mathrm{IH}$ systems working close to resonance contain more than $95 \%$ of the power in the first harmonic [24]. Therefore, in terms of power, high harmonics are negligible.

\section{B. Current through the inductors}

The prediction of the current flowing through the coils is a key issue as it determines the design of the converter. For this reason, the simulated current waveforms through the coils will be verified by the measuring the currents through the inductors.

In this case, higher harmonics take greater importance as they determine important factors as the peak current and the turn off current. Therefore, equations (6) and (7) have

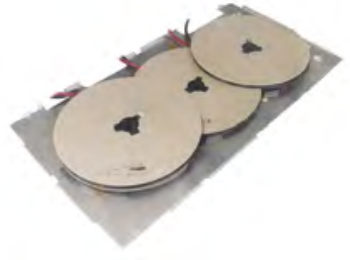

(a)

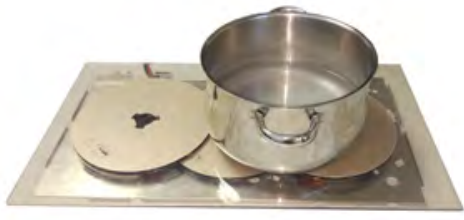

(b)
Fig. 12: IH coil array prototype. (a) Unloaded system. (b) Loaded system.

been applied for a large number of harmonics. The results are compared with the measurements in Fig. 14, where measured currents are shown in red, and calculated currents are represented in black. These are the sum of the most relevant harmonics, which are shown in blue. In this case, the half-bridge inverter supplying to inductor 1 has a duty cycle of $D_{1}=0.26$ and inductor 2 is being powered by a square voltage $\left(D_{2}=0.5\right)$. Both inverters have a switching frequency of $40 \mathrm{kHz}$.

As can be seen, the model correctly predicts the current waveforms. The experimental results are coherent with other similar systems in the literature [25]-[29]. In addition, the model replicates the effect of the phase-shift between the inverter's output voltages. We can observe that in field addition (inductors in phase), the better coupling to the vessel produces an equivalent larger impedance and therefore lower currents. On the other hand, coils in field cancellation (inductors in counter-phase) are less coupled to the pot, which reduces the equivalent impedance and generates larger currents.

\section{Surface power density}

Surface power density cannot be measured directly, however, the temperature in the base of the pot gives a fair indication of whether power is being correctly distributed, or on the contrary, hot and cold spots are being generated.

A ferromagnetic pot with a diameter of $28 \mathrm{~cm}$ was painted in black and examined by a FLIR AX8 thermographic camera when being heated. The tests were run with the empty vessel at $1 \mathrm{~kW}$. Fig. 15 compares the simulated power density $p_{\text {pot }}$ with the measured temperature in the base of the pot after 10 seconds. The experiments were run in-phase, in counter-phase and in an in-between phase shift with equal current amplitudes in both coils. The temperature measurements verify the effect of the coupling in the surface power density distribution, however, the temperature in the base of the pot is uneven. Simulations show a slight power density difference between coils 1 and 2 (top layer and bottom layer respectively) mainly due to the different distance to the load, but also influenced by the inherent asymmetric power density distribution when using of coupled inductors at in-between phase-shifts [30], [31]. On the other hand, the difference in terms of temperature 


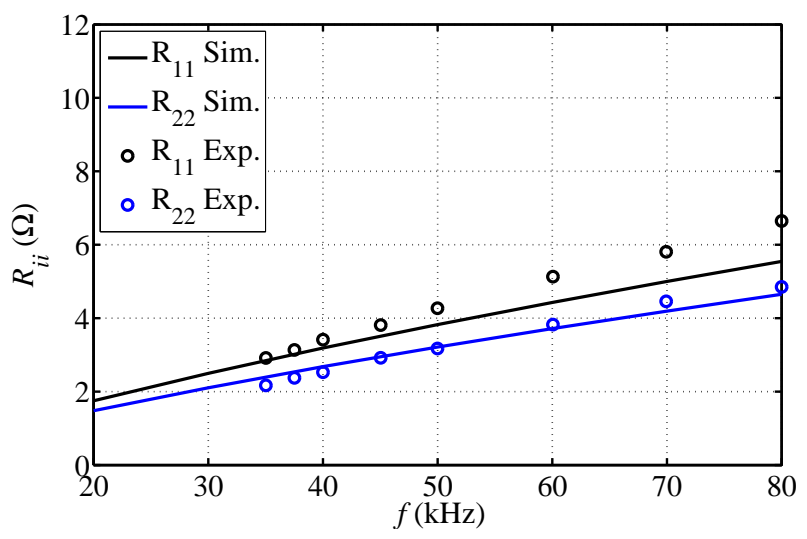

(a)

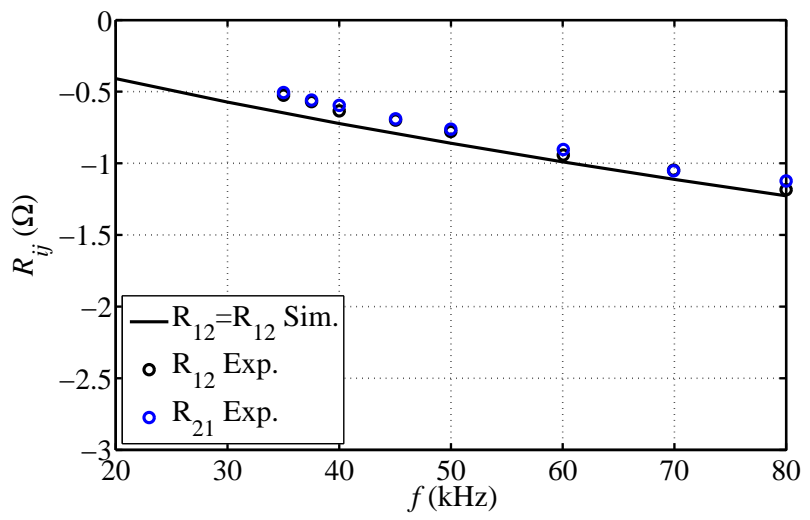

(c)

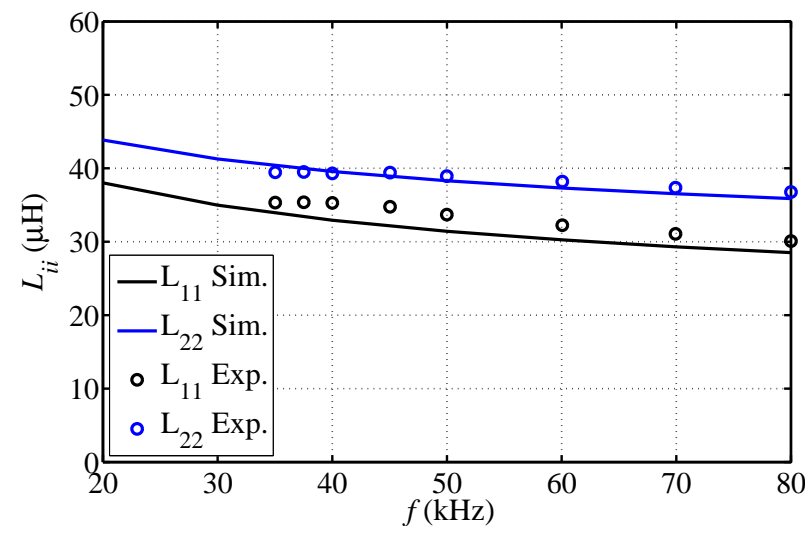

(b)

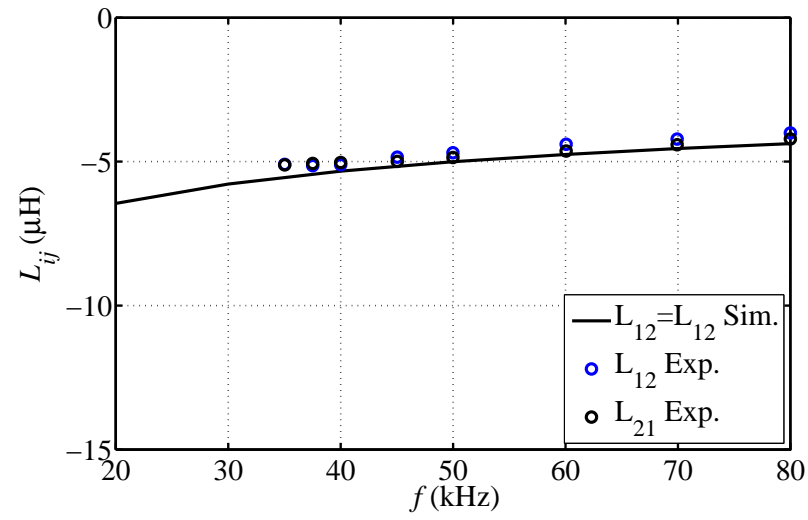

(d)

Fig. 13: Simulated (solid lines) and measured (circles) first harmonic impedance matrix. (a) $R_{i i}$. (b) $L_{i i}$. (c) $R_{i j}$. (d) $L_{i j}$.

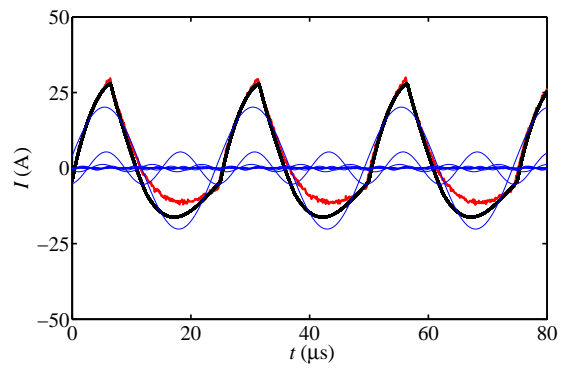

(a)

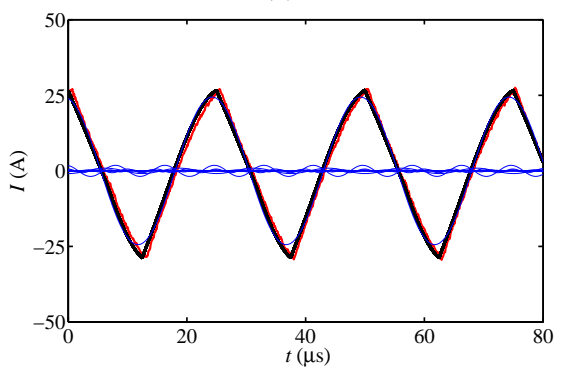

(d)

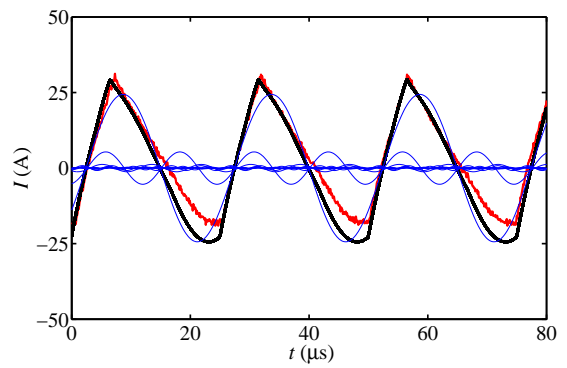

(b)

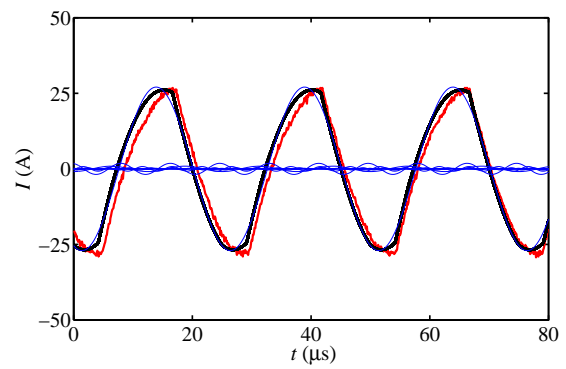

(e)

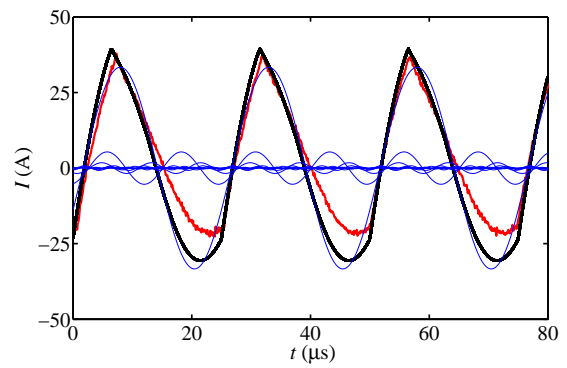

(c)

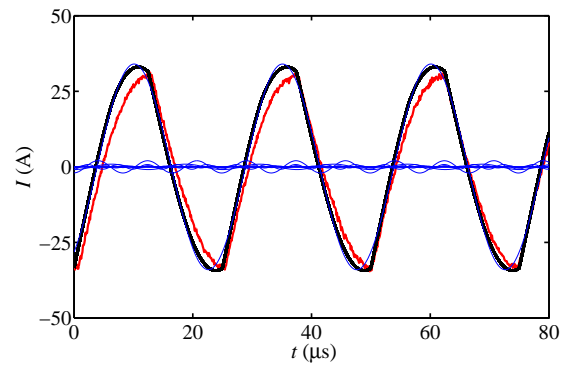

(f)

Fig. 14: Current through the inductors when applying an asymmetrical duty cycle modulation to inductor $1\left(D_{1}=0.26\right)$ and square wave modulation to inductor $2\left(D_{1}=0.5\right)$. Measured in red, simulated in black, harmonic content in blue. (a) $I_{1}$ with inverters in phase $\left(\varphi_{v}=0^{\circ}\right)$. (b) $I_{1}$ applying an in-between phase $\left(\varphi_{v}=120^{\circ}\right)$. (c) $I_{1}$ with inverters in counter-phase $\varphi_{v}=180^{\circ}$. (d) $I_{2}$ with inverters in phase $\left(\varphi_{v}=0^{\circ}\right)$. (e) $I_{2}$ applying an in-between phase $\varphi_{v}=120^{\circ}$. (f) $I_{2}$ with inverters in counter-phase $\left(\varphi_{v}=180^{\circ}\right)$. 


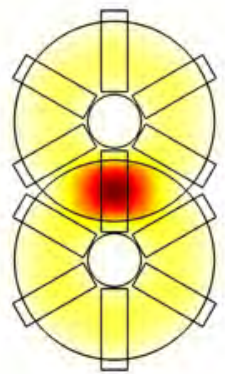

(a)

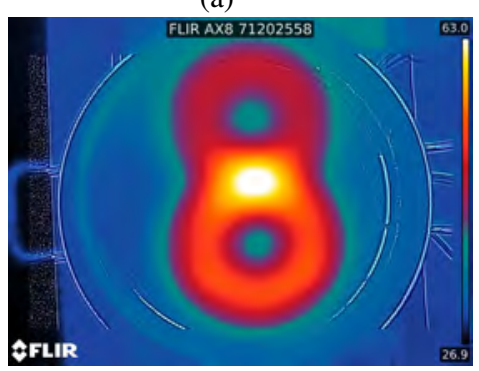

(d)

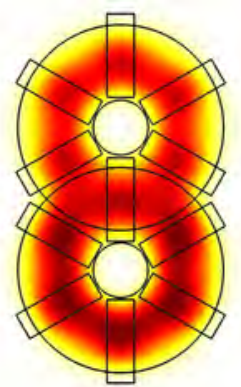

(b)

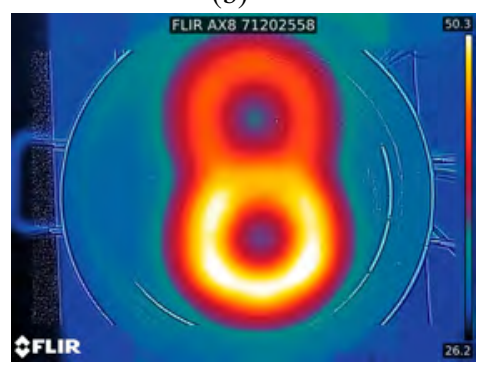

(e)

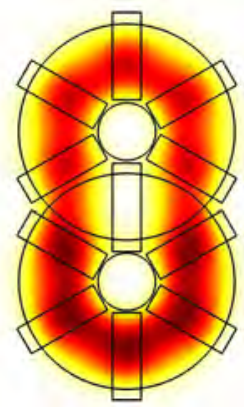

(c)

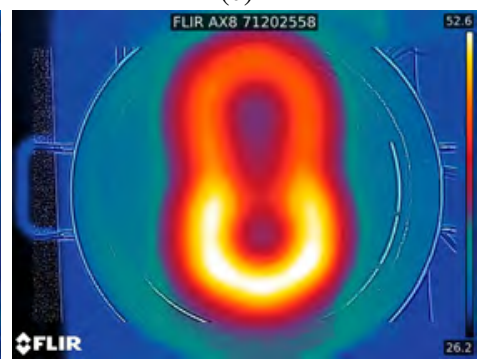

(f)

Fig. 15: Comparison between simulated surface power density distribution and measured temperature when the same current is flowing through each coil. Simulated $p_{\text {pot }}$ : (a) In-phase. (b) In-between phase. (c) Counter-phase. Measured temperature: (d) In-phase. (e) In-between phase. (f) Counter-phase.

is significant. As the magnetic field is proportional to product of the number of turns and the driven current, the temperature measurements indicate that the coil in the lower layer needs either a higher current or a larger number of turns to produce an even temperature distribution.

\section{CONCLUSIONS}

In this work, the technique of overlapped inductors, commonly used in WPT systems, has been evaluated for the application of domestic IH. This is an innovative approach in the search for efficient flexible cooking areas, as allows the use of large inductors with high performance while maintaining the pot adaptability in terms of size, shape and position. The system has been both theoretically studied and experimentally characterized.

The proposed configuration presents several advantages. Firstly, each of the inductors is able to supply to the pot up to $3.7 \mathrm{~kW}$ with a very high efficiency, which is around $98 \%$ while small inductors in flexible cooking areas have typically efficiencies of $96 \%$. In the second place, the number of inductors is reduced together with their accessory elements as ferrites and mounting elements. Moreover, the number of inverters can be also reduced. This implies a significant cost reduction.

On the other hand, the systems presents some drawbacks mainly caused by the fact that inductors have a different distance to the pot, and therefore, different impedance. The main consequences are a hampered power control and an uneven surface power distribution, needing corrective strategies as injecting more current in the bottom layer coil or designing this coil with a greater number of turns to increase the magnetic field.

However, the study proves the viability of overlapped coils for IH appliances and throws promising results, opening a novel developing area in domestic induction heating appliances. The simulation model correctly predicts the behavior of the prototype, and therefore constitutes a powerful tool for further improvement.

\section{REFERENCES}

[1] W. C. Moreland, "The induction range: Its performance and its development problems," IEEE Transactions on Industry Applications, vol. IA-9, no. 1, pp. 81-85, Jan. 1973.

[2] O. Lucía, J. Acero, C. Carretero, and J. Burdío, "Induction heating appliances: Toward more flexible cooking surfaces," IEEE Ind. Electron. Mag., vol. 7, no. 3, pp. 35-47, Sep. 2013.

[3] J. Acero, C. Carretero, R. Alonso, and J. M. Burdio, "Quantitative evaluation of induction efficiency in domestic induction heating applications," IEEE Transactions on Magnetics, vol. 49, no. 4, pp. 1382-1389, Apr. 2013.

[4] C. Carretero, R. Alonso, J. Acero, and J. M. Burdio, "Optimized 4coil inductor system arrangement for induction heating appliances," in IECON 2015 - 41st Annual Conference of the IEEE Industrial Electronics Society, pp. 004 948-004 952, Nov. 2015.

[5] G. A. Covic and J. T. Boys, "Inductive power transfer," Proc. IEEE, vol. 101, no. 6, pp. 1276-1289, Jun. 2013.

[6] G. A. Covic and J. T. Boys, "Modern trends in inductive power transfer for transportation applications," IEEE Journal of Emerging and Selected Topics in Power Electronics, vol. 1, no. 1, pp. 28-41, Mar. 2013.

[7] S. Kim, A. Tejeda, G. A. Covic, and J. T. Boys, "Analysis of mutually decoupled primary coils for IPT systems for EV charging," in 2016 IEEE Energy Conversion Congress and Exposition (ECCE), pp. 1-6, Sep. 2016. 
[8] Y. Li, R. Mai, L. Lu, T. Lin, Y. Liu, and Z. He, "Analysis and transmitter currents decomposition based control for multiple overlapped transmitters based WPT systems considering cross couplings," IEEE Transactions on Power Electronics, vol. PP, no. 99, pp. 1-1, 2017.

[9] G. A. J. Elliott, S. Raabe, G. A. Covic, and J. T. Boys, "Multiphase pickups for large lateral tolerance contactless power-transfer systems," IEEE Transactions on Industrial Electronics, vol. 57, no. 5, pp. 15901598, May. 2010.

[10] K. R. Davey and D. Zheng, "Prediction and use of impedance matrices for eddy-current problems," IEEE Transactions on Magnetics, vol. 33, no. 4, pp. 2478-2485, Jul. 1997.

[11] E. E. Mombello, "Impedances for the calculation of electromagnetic transients within transformers," IEEE Transactions on Power Delivery, vol. 17, no. 2, pp. 479-488, Apr. 2002.

[12] I. Lope, J. Acero, and C. Carretero, "Analysis and optimization of the efficiency of induction heating applications with litz-wire planar and solenoidal coils," IEEE Transactions on Power Electronics, vol. 31, no. 7, pp. 5089-5101, Jul. 2016.

[13] Ferroxcube, "Datasheet 3C90 material specification," Tech. Rep., 2008.

[14] M. Adler, "A field-theoretical approach to magnetic induction heating of thin circular plates," IEEE Transactions on Magnetics, vol. 10, no. 4 pp. 1118-1125, Dec. 1974.

[15] C. Carretero, J. Acero, R. Alonso, and J. M. Burdio, "Normal-mode decomposition of surface power distribution in multiple-coil induction heating systems," IEEE Transactions on Magnetics, vol. 52, no. 2, pp. 1-8, Feb. 2016

[16] J. Ferreira, "Analytical computation of ac resistance of round and rectangular litz wire windings," Electric Power Applications, IEE Proceedings $B$, vol. 139 , no. 1 , pp. 21-25, Jan. 1992.

[17] J. Ferreira, "Improved analytical modeling of conductive losses in magnetic components," IEEE Trans. Power Electron., vol. 9, no. 1, pp. 127-131, Jan. 1994.

[18] C. Sullivan, "Optimal choice for number of strands in a litz-wire transformer winding," IEEE Trans. Power Electron., vol. 14, no. 2, pp. 283-291, Mar. 1999.

[19] H. Hamalainen, J. Pyrhonen, J. Nerg, and J. Talvitie, "Ac resistance factor of litz-wire windings used in low-voltage high-power generators," IEEE Trans. Ind. Electron., vol. 61, no. 2, pp. 693-700, Feb. 2014.

[20] J. Acero, R. Alonso, J. Burdío, L. Barragán, and D. Puyal, "Frequencydependent resistance in litz-wire planar windings for domestic induction heating appliances," IEEE Trans. Power Electron., vol. 21, no. 4, pp. 856-866, Jul. 2006.

[21] C. Carretero, "Coupling power losses in inductive power transfer systems with litz-wire coils," IEEE Transactions on Industrial Electronics, vol. 64, no. 6, pp. 4474-4482, Jun. 2017.

[22] C. Carretero, J. Acero, and R. Alonso., "TM-TE decomposition of power losses in multi-stranded litz-wires used in electronic devices," Progress In Electromagnetics Research, vol. 123, pp. 83-103, Dec. 2012.

[23] D. Puyal, C. Bernal, J. M. Burdio, J. Acero, and I. Millan, "Versatile high-frequency inverter module for large-signal inductive loads characterization up to $1.5 \mathrm{mhz}$ and $7 \mathrm{kw}$," IEEE Transactions on Power Electronics, vol. 23, DOI 10.1109/TPEL.2007.911854, no. 1, pp. 7587, Jan. 2008

[24] F. Forest, S. Faucher, J.-Y. Gaspard, D. Montloup, J.-J. Huselstein, and C. Joubert, "Frequency-synchronized resonant converters for the supply of multiwinding coils in induction cooking appliances," IEEE Trans. Ind. Electron., vol. 54, DOI 10.1109/TIE.2006.888797, no. 1, pp. 441-452, Feb. 2007.

[25] F. Forest, E. Labouré, F. Costa, and J. Gaspard, "Principle of a multiload/single converter system for low power induction heating," IEEE Trans. Power Electron., vol. 15, DOI 10.1109/63.838094, no. 2, pp. 223-230, Mar. 2000.

[26] H. Sarnago, O. Lucia, and J. M. Burdio, "Interleaved resonant boost inverter featuring sic module for high-performance induction heating," IEEE Transactions on Power Electronics, vol. 32, DOI 10.1109/TPEL.2016.2554607, no. 2, pp. 1018-1029, Feb. 2017

[27] N. Lecic, G. Stojanovic, S. Djuric, and E. Laboure, "Design and analysis of planar symmetric six-phase coupled inductors," IEEE Transactions on Magnetics, vol. 51, DOI 10.1109/TMAG.2014.2383358, no. 6, pp. 1-8, Jun. 2015

[28] T. Mishima, S. Sakamoto, and C. Ide, "Zvs phase-shift pwm-controlled single-stage boost full-bridge ac-ac converter for high-frequency induction heating applications," IEEE Transactions on Industrial Electronics,

[29] C. Bi, H. Lu, K. Jia, J. Hu, and H. Li, "A novel multiple-frequency resonant inverter for induction heating applications," IEEE Transactions on vol. 64, DOI 10.1109/TIE.2016.2620098, no. 3, pp. 2054-2061, Mar. 2017.

Power Electronics, vol. 31, DOI 10.1109/TPEL.2016.2521401, no. 12 , pp. 8162-8171, Dec. 2016.

[30] S. Lupi, M. Forzan, F. Dughiero, and A. Zenkov, "Comparison of edge-effects of transverse flux and travelling wave induction heating inductors," IEEE Transactions on Magnetics, vol. 35, DOI 10.1109/20.800588, no. 5, pp. 3556-3558, Sep. 1999.

[31] L. Pang, Y. Wang, and T. Chen, "New development of traveling wave induction heating," IEEE Transactions on Applied Superconductivity, vol. 20, DOI 10.1109/TASC.2010.2040378, no. 3, pp. 1013-1016, Jun. 2010.

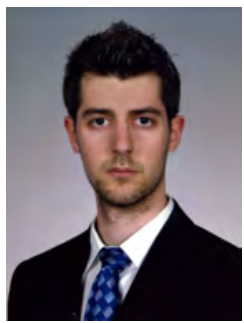

Javier Serrano (S'15) received the M.Sc. degree in Industrial Engineering from the University of Zaragoza, Spain, in 2015, where he is currently working toward the Ph.D. degree in Power Electronics in the Department of Electronic Engineering and Communications. His main research interests include electromagnetic modeling of magnetic devices, modeling of power losses in high-frequency applications and characterization of induction heating systems.

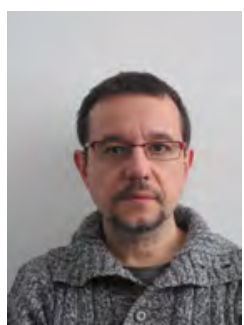

Jesús Acero (M'06) received the M.Sc. and $\mathrm{Ph}$.D. degrees in electrical engineering from the University of Zaragoza, Spain, in 1992 and 2005 , respectively, where he is currently an Associate Professor. His main research interests include resonant converters for induction heating applications, inductive-type load modeling, and magnetics. Dr. Acero is since 2015 Associate Editor of the IEEE Transactions on Power Electronics. Dr. Acero is a member of the Aragon Institute for Engineering Research (I3A).

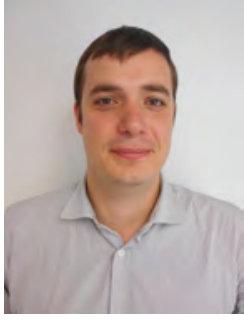

Ignacio Lope (M'16) received the M.Sc. degree in Electrical Engineering and the Ph.D. in Power electronics from the University of Zaragoza, Spain, in 2010 and 2015. He is currently with the BSH Home Appliances Spain involved in several projects focused on developing domestic induction heating appliances. His current research interests include electromagnetic modeling of inductive coupled contactless energy transfer systems, and loss modeling of magnetic devices.

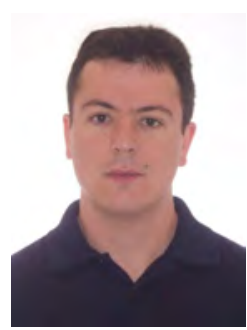

Claudio Carretero (SM'15) received the B.Sc and M.Sc. degree in physics, the B.Sc. and M.Sc. degree in electrical engineering and the $\mathrm{Ph} . \mathrm{D}$. degree in electrical engineering from the University of Zaragoza, in 1998, 2002 and 2010, respectively. $\mathrm{He}$ is currently Assistant Professor at the Department of Applied Physics, University of Zaragoza. His research interests include induction heating applications and electromagnetic modeling of inductive systems. Dr. Carretero is a member of the Dr. Carretero is a member of the Aragon Institute for Engineering Research (I3A).

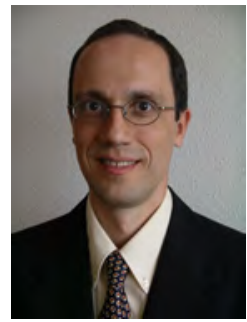

Jose Miguel Burdío (SM'13) received the M.Sc. and $\mathrm{Ph} . \mathrm{D}$. degrees in electrical engineering from the University of Zaragoza, Zaragoza, Spain, in 1991 and 1995, respectively. He is with the Department of Electronic Engineering and Communications, University of Zaragoza, where he is currently a Professor. His main research interests include modeling of switching converters and resonant power conversion for inductionheating applications. Dr. Burdío is a member of the Aragon Institute for Engineering Research 